\title{
Analysis of Polyphenols in the Lamiaceae Family by Matrix Solid- Phase Dispersion Extraction Followed by Ultra-High-Performance Liquid Chromatography-Tandem Mass Spectrometry Determination
}

\author{
Maria Chiara Simeoni, Marika Pellegrini, Manuel Sergi,*ib Paola Pittia, Antonella Ricci, \\ and Dario Compagnone \\ Faculty of Bioscience and Technologies for Food, Agriculture and Environment, University of Teramo, Via Renato Balzarini, 1, \\ 64100 Teramo, Italy
}

Supporting Information

\begin{abstract}
Polyphenolic compounds (PCs) are natural-occurring secondary metabolites with considerable physiological and morphological importance in plants and different biological functions. In this work, a matrix solid-phase dispersion extraction/purification procedure, followed by an ultra-highperformance liquid chromatography-tandem mass spectrometry analysis, has been developed and validated for the quantification of 17 PCs in Ocimum basilicum L., Origanum vulgare L., and Thymus vulgaris L. from the Lamiaceae family. The use of a limited sample amount, combined with small solvent consumption, marks the convenience of this technique for the extraction/ isolation of compounds of interest. The validation showed good results, with recoveries ranged between 75 and $105 \%$, relative standard deviation values $\leq 12 \%$, and very low matrix effects. The experimental results demonstrated the variability of the phenolic pattern of the samples and the need for accurate assessment of the phenolic pattern to establish the real nutraceutical proprieties of food products.
\end{abstract}

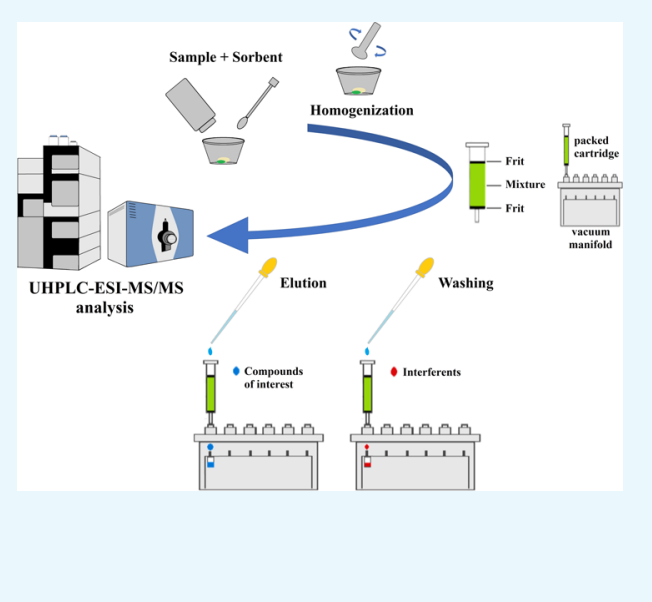

\section{INTRODUCTION}

Plants belonging to the Lamiaceae family are well-known for their high content of polyphenolic compounds (PCs), naturaloccurring secondary metabolites with a large variety of structures. ${ }^{1}$ These compounds possess significant morphological and physiological relevance in plants and, as a huge group of bioactive compounds, they possess different biological functions; PCs, in fact, can act as phytoalexins, ${ }^{2}$ possess antioxidant properties, contribute to plant pigmentation, and have the ability to attract pollinators and protect plants from UV radiation, insects, and grazing animals. ${ }^{3}$ These properties allow PCs to contribute to plant development, reproduction, inhibition of pathogens and predators, as well as color and sensory features of fruits and vegetables. ${ }^{4}$ The accumulation of these molecules in plants is influenced by different aspects such as cultivation methods, cultivar, growth and maturation, besides conditions of storage and process. ${ }^{3}$

During the last century, PCs have been widely studied for their potentiality in prevention and treatment of oxidative stress-related diseases; several in vitro and in vivo studies exposed their involvement in the prevention of several diseases, such as cardiovascular and some neurodegenerative illness, cancers, osteoporosis, and diabetes mellitus. ${ }^{5,6}$ These compounds are also exploited in several industrial fields, as pigments and preservatives in the food industry; as paints and paper in the manufacturing sector; and as natural additives in cosmetics. For all these reasons, significant efforts have been made in the characterization of phenolics of different vegetal tissues. $^{4}$

Antioxidant capacity of polyphenols is one of the key issues to understand their role in health and food systems; this can be rapidly assessed using classical spectrophotometric assays or newly proposed colorimetric or sensor-based approaches. ${ }^{7}$ These assays measure the total antioxidant capacity of the entire phenolics or can give an idea of the prevalence of some classes of compounds (i.e., $o$-diphenols vs monophenols)., Plant phenolics can be divided into flavonoids, tannins, phenolic acids, and the less common stilbenes and lignans. Flavonoids present a basic flavan nucleus structure with 15 carbon atoms arranged in three rings (C6-C3-C6) (labeled as $\mathrm{A}, \mathrm{B}$, and $\mathrm{C}$ ). According to the oxidation state of the central $\mathrm{C}$ ring, flavonoids can be classified into six subgroups: anthocyanins, flavanols, flavanones, flavones, flavonols, and isoflavones. Tannins have an important role in human diet and can be usually subdivided into hydrolysable tannins and condensed tannins. ${ }^{4}$ Phenolic acids are regrouped into

Received: August 31, 2018

Accepted: November 14, 2018

Published: December 18, 2018 


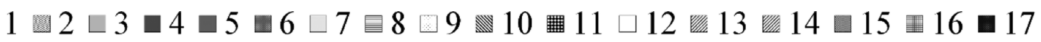

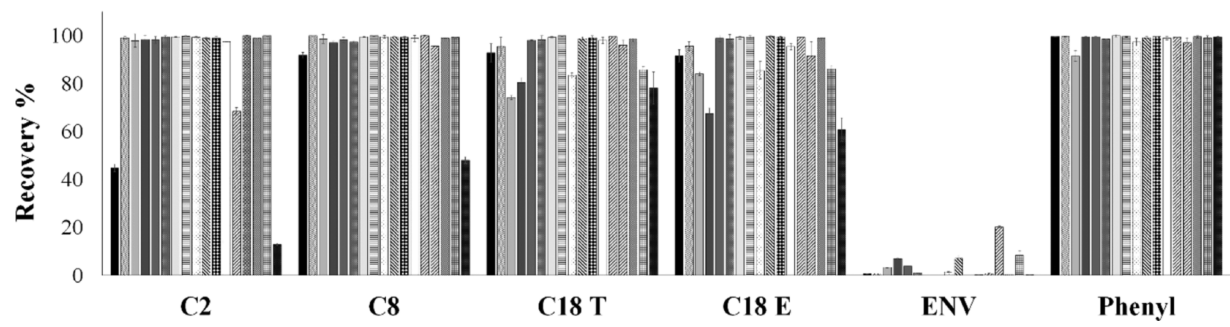

Figure 1. Recovery \% obtained for the first elution step of each different phase tested: C2, C8, C18 T (uncapped), C18 E (capped), PHENYL, and ENV. In the figure: 1-gallic acid; 2-caftaric acid; 3-chlorogenic acid; 4-4-OH phenylacetic acid; 5-4-OH benzoic acid; 6-caffeic acid; 7isoquercetin; 8-vanillic acid; 9-syringic acid; 10 - chicoric acid; $11-p$-coumaric acid; 12 -ferulic acid; 13-rutin; 14-rosmarinic acid; 15-ocoumaric acid; 16-cinnamic acid; 17-quercetin.

derivatives of benzoic acid (e.g., gallic acid) and derivatives of cinnamic acid (e.g., caffeic, coumaric, and ferulic acid). ${ }^{10}$

The PCs mainly found in previous studies in the Lamiaceae family plant are caffeic acid, chicoric acid, cinnamic acid, quercetin, and rosmarinic acid. ${ }^{11-13}$ Their separation and determination are commonly achieved through different chromatographic techniques coupled to different detection strategies, such as high-performance liquid chromatography (HPLC)-diode array detection, ${ }^{14,15}$ gas chromatographyelectron ionization-mass spectrometry (MS), ${ }^{16,17}$ HPLCtandem MS (MS/MS), ${ }^{18,19}$ ultra-HPLC (UHPLC)-MS/ MS. $^{20,21}$

Despite the wide range of possible chromatographic separation and detection couplings, the resulting phenolic pattern is extremely dependent on the extraction procedure. Extraction efficiency is deeply influenced by the chemical composition of the sample, particle size, extraction solvent, and method employed, as well as by the presence of interfering compounds.

Matrix solid-phase dispersion (MSPD) is an analytical method developed in 1989 for the drug and metabolite residues' isolation from tissues. ${ }^{22}$ Since its first description, this technique has been reported as the extraction method in several publications ${ }^{23}$ for the isolation of a widespread range of molecules from different plant and animal samples. The chemical and physical principles of MSPD involve (i) mechanical blending forces, for the whole sample disruption; (ii) sample matrix interactions with a solid support chemically derivatized; (iii) isolation of the target analytes through hydrophobic and hydrophilic interactions; (iv) application of a solvent, or a sequence of solvents, to eluate the compounds of interest.

This technique is suitable for complex matrices and any biological material and heterogeneous samples, such as mycotoxins and polyphenols, ${ }^{23,24}$ naturally occurring constituents in plants ${ }^{25}$ and xenobiotics in animal tissues. ${ }^{26}$ Recently, an MSPD procedure for seven PCs in dried olive fruits has been proposed. ${ }^{27}$

In this work, an MSPD extraction procedure, followed by a UHPLC-MS/MS analysis, has been developed for the quantitative characterization of 17 PCs. The application of the method to Ocimum basilicum L., Origanum vulgare L., and Thymus vulgaris L. from the Lamiaceae family demonstrated the variability of the phenolic pattern of the samples and the need for accurate assessment of the phenolic pattern to establish the real nutraceutical proprieties of food products.

\section{RESULTS AND DISCUSSION}

MSPD Extraction Optimization. PCs are secondary metabolites widely present in plants with variable concentrations and structures. The organic compounds belonging to this family, in fact, can be from simple molecules (e.g., anthocyanins, phenolic acids) to highly polymerized complexes (e.g., tannins) or can be found in association with other plant compounds, such as proteins or saccharides.

In this work, a reliable MSPD procedure has been developed with the aim of achieving the best PC extraction conditions, which also allow to minimize the matrix effect because of several matrix components.

The first step was the selection of the suitable dispersing phase in order to obtain the best recovery rates coupled with good selectivity. The minimum eluent volume able to get satisfying recoveries for all analytes was also investigated. At the beginning, the tests were carried out with standard solutions of the analytes, using methanol as elution solvent as it has been generally found to be effective in extraction of polyphenols with low molecular weights. The following phases have been tested: C2, C8, C18 (capped and uncapped), phenyl as silica-based sorbent, and a polymeric phase (crosslinked hydroxylated polystyrene-divinylbenzene copolymer) ENV. ENV did not show a linear behavior toward the analytes; so, it was excluded from further experiments. The best extraction results were obtained with $\mathrm{C} 18$ uncapped $(\mathrm{C} 18 \mathrm{~T})$ and phenylbonded phases that led to significant recoveries with just 500 $\mu \mathrm{L}$ of eluent volume (Figure 1).

The complexity of the plant matrix and the fact that the MSPD sorbent retains not only analytes, but also other nonpolar molecules, requires a washing step to minimize the interferences from unrelated substances such as sugar, organic acids, and fats. Therefore, a supplementary step is necessary to remove as much as possible these unwanted compounds and their potential co-elution with phenolic acids.

Therefore, extraction tests with $O$. basilicum samples were carried out using $\mathrm{C} 18 \mathrm{~T}$ and phenyl as MSPD sorbents; the suitable sample/matrix ratio able to achieve the best extraction efficiency was a 1:3 ratio, confirmed also by matrix effects experiments reported in the Validation Results section. To obtain a selective removal of interfering compounds without significant loss of analytes, washing solutions with various ratios of water/methanol $(90: 10,75: 25,60: 40 \mathrm{v} / \mathrm{v})$ have been tested. In order to evaluate the amount of analyte loss in the washing step, the washing discharges were collected and analyzed: for both phases, an amount of methanol higher than $10 \%$ led to a significant loss of analytes, especially the most 
A

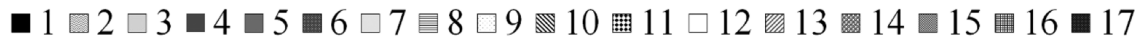
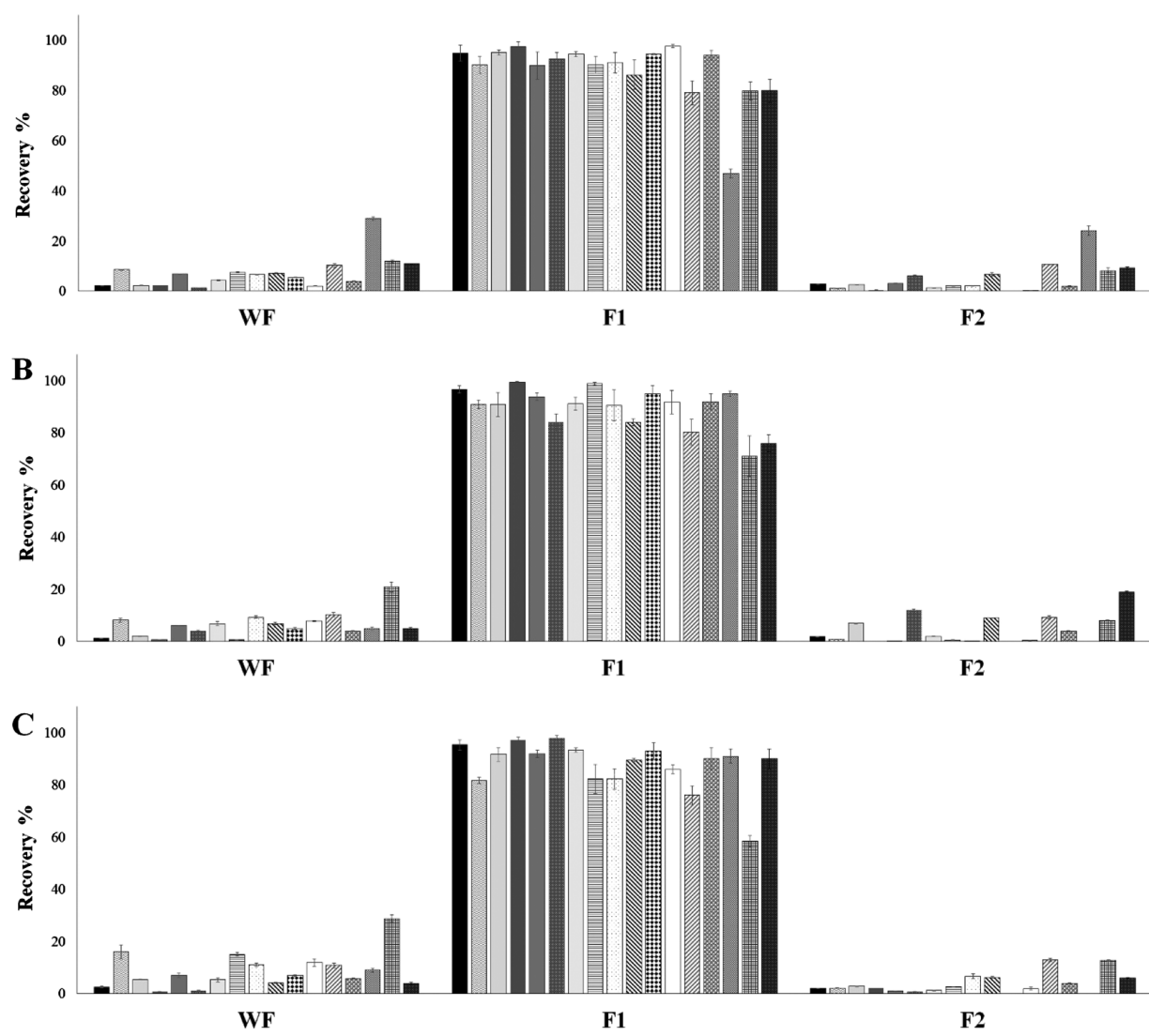

Figure 2. Recovery \% calculated for different water/methanol washing solutions 90:10 (A); 75:25 (B); 60:40 (C). In the figure: WF-wash fraction, F1-first elution, F2-second elution; 1-gallic acid; 2-caftaric acid; 3-chlorogenic acid; 4-4-OH phenylacetic acid; 5-4-OH benzoic acid; 6-caffeic acid; 7-isoquercetin; 8-vanillic acid; 9-syringic acid; 10-chicoric acid; 11-p-coumaric acid; 12-ferulic acid; 13rutin; 14-rosmarinic acid; 15-o-coumaric acid; 16-cinnamic acid; 17-quercetin.

polar ones; therefore, $1 \mathrm{~mL}$ of a solution of water/methanol 90:10 v/v was chosen (Figure 2).

To evaluate the best dispersing media for MSPD between $\mathrm{C} 18 \mathrm{~T}$ and phenyl phases, their performances were compared in terms of recovery, matrix effect, and reproducibility. As the extraction efficiency is comparable between the two selected sorbents, the $\mathrm{C} 18$ phase was chosen as phenyl gave problems in the washing step, with loss of analytes even with the 90:10 water/methanol solution.

UHPLC-MS/MS Optimization. In LC-MS/MS analysis of phenolic compounds, with quite different polarities, the chromatographic conditions must be optimized carefully to obtain a good separation of all compounds and a suitable retention for the most polar ones.

For this purpose, different chromatographic columns were tested: from classic C18 $(100 \times 2.10 \mathrm{~mm}$ from Phenomenex $)$ packed with $1.7 \mu \mathrm{m}$ core shell particles, to polar phases as OBELISC R $(150 \times 2.10 \mathrm{~mm}$ from SIELC) with particle size of $5 \mu \mathrm{m}$; also, a mixed-phase C18-PFP Excel $2(10 \mathrm{~cm} \times 2.1$ $\mathrm{mm}$ from ACE) packed with particles of $2 \mu \mathrm{m}$. Of course, also different mobile phases combinations, with several amounts of formic acid and acetic acid, were tested.

Obelisc R exhibited good retention for polar compounds, but poor separation, whereas $\mathrm{C} 18$ provided reliable features, especially for more apolar compounds; anyway, the mixed C18-PFP stationary phase improved the retention of polar compounds and led to excellent performances both in separation and in efficiency for all analytes. As organic mobile phase, acetonitrile gave better performances than methanol; moreover, the formic acid addition in water resulted in an enhancement of the signals, whereas acetic acid led to a lowering of electrospray ionization (ESI). An aqueous $0.1 \%$ formic acid phase was the best compromise to get good intensity and shape of peaks.

Two precursor ion/product ion transitions were chosen for each analyte. Ion currents' acquisition was carried out in multireaction monitoring (MRM) mode. For the selected analytes, source and instrument parameters were optimized through syringe pump injection of each single standard methanolic solution $\left(10 \mathrm{ng} \mathrm{mL} L^{-1}\right)$ at a flow of $10 \mu \mathrm{L} \mathrm{min}^{-1}$.

Validation Results. The validation data show good analytical performances in terms of repeatability, linearity recoveries, and matrix effect. Good linearity was obtained for each analyte, with correlation coefficients $\left(r^{2}\right)>0.999$ in a wide range of concentrations.

The results displayed a minimal matrix effect, both qualitatively and quantitatively, so the combination of a suitable washing solution, a very apolar sorbent phase as C18T and a polar elution solvent as methanol, allowed the selective extraction of PCs in the selected vegetal matrices. The analysis of matrix behavior and the comparison with that of mobile phase reference led to the evaluation of ion suppression effect 
qualitatively. As shown in Figure S1 (see the Supporting Information), there is no other ion suppression area recognized in the chromatograms that could affect the ion signal of our analytes; the only area of ion suppression is in the first part of the LC chromatogram, before the retention time of gallic acid, the first eluted analyte. Besides MSPD extraction and MS/MS detection, the improved retention of the polar analytes, provided by the C18-PFP column, especially gallic acid, allows to obtain further selectivity to the whole method, by removing chromatographically the potential interfering compounds from the acquisition of analyte signal.

Moreover, the method showed highly acceptable analytical precisions with relative standard deviation (RSD) values $\leq 12 \%$ (intraday precision or repeatability) and $\leq 10 \%$ (interday precision or within-laboratory reproducibility). All validation parameters, including limit of detection (LOD) and limit of quantification (LOQ) for each compound, are reported in Tables $1-3$.

Table 1. Limits of Quantitation and Detection for Each Compound

\begin{tabular}{lccc}
\multicolumn{1}{c}{ compound } & LOQ $\left(\mathrm{ng} \mathrm{g}^{-1}\right)$ & LOD $\left(\mathrm{ng} \mathrm{g}^{-1}\right)$ & $r^{2}$ \\
gallic acid & 3.00 & 0.90 & 0.9999 \\
caftaric acid & 15.00 & 4.50 & 0.9997 \\
chlorogenic acid & 0.30 & 0.09 & 0.9990 \\
4-OH benzoic acid & 0.90 & 0.30 & 0.9997 \\
4-OH phenylacetic acid & 5.00 & 1.80 & 0.9996 \\
isoquercetin & 0.60 & 0.18 & 0.9998 \\
caffeic acid & 1.50 & 0.48 & 0.9996 \\
vanillic acid & 0.60 & 0.18 & 0.9995 \\
syringic acid & 3.00 & 0.90 & 0.9994 \\
$p$-coumaric acid & 1.20 & 0.45 & 0.9998 \\
ferulic acid & 1.80 & 0.60 & 0.9999 \\
chicoric acid & 0.18 & 0.06 & 0.9997 \\
rutin & 0.15 & 0.06 & 0.9997 \\
$o$-coumaric acid & 0.30 & 0.09 & 0.9997 \\
rosmarinic acid & 1.50 & 0.48 & 0.9995 \\
cinnamic acid & 4.50 & 1.50 & 0.9998 \\
quercetin & 0.30 & 0.09 & 0.9999 \\
\hline
\end{tabular}

Table 2. Repeatability and Reproducibility Obtained for Each Compound

\begin{tabular}{lcc}
\multicolumn{1}{c}{ compound } & intraday RSD \% & interday RSD \% \\
gallic acid & 7 & 11 \\
caftaric acid & 10 & 8 \\
chlorogenic acid & 4 & 9 \\
4-OH benzoic acid & 3 & 6 \\
4-OH phenylacetic acid & 9 & 3 \\
isoquercetin & 5 & 7 \\
caffeic acid & 11 & 6 \\
vanillic acid & 8 & 2 \\
syringic acid & 5 & 7 \\
$p$-coumaric acid & 12 & 10 \\
ferulic acid & 8 & 8 \\
chicoric acid & 7 & 3 \\
rutin & 10 & 9 \\
$o$-coumaric acid & 11 & 7 \\
rosmarinic acid & 5 & 3 \\
cinnamic acid & 12 & 10 \\
quercetin & 8 & 6
\end{tabular}

Real Samples. Samples were subjected to the entire method; the quantitation of each targeted phenolic compound was performed through the regression curves obtained via the standard addition method. The PC amounts in real samples are reported in Table 4.

\section{CONCLUSIONS}

The results of this study have allowed the optimization of an MSPD extraction method using a C18 sorbent for the isolation of PCs present in vegetal matrices, followed by UHPLC-MS/ MS analysis. MSPD with a C18 silica base as dispersing phase, a suitable washing solution, and methanol as elution solvent allow a selective extraction of PCs. The validation parameters showed good reproducibility of the method with suitable sensitivity and specificity, as proved by low levels of the matrix effect; so, this method could be a useful tool for the evaluation of PC content in vegetal samples.

\section{GENERAL EXPERIMENTAL PROCEDURES}

Standards and Reagents. Gallic acid, caftaric acid, chlorogenic acid, 4-OH benzoic acid, 4-OH phenylacetic acid, vanillic acid, syringic acid, $p$-coumaric and $o$-coumaric acids, ferulic acid, chicoric acid, rutin, rosmarinic acid, cinnamic acid, quercetin (from Sigma-Aldrich, Milan, Italy), isoquercetin, and caffeic acid (from Extrasynthese S.A., Genay, France) standards were employed; working standard mixtures were prepared by appropriate dilution of the standards in methanol. All solutions were stored at $-20{ }^{\circ} \mathrm{C}$ in the dark. Ultrapure water, formic acid, methanol, and acetonitrile used were UPLC-MS grade and were purchased from Carlo Erba (Milan, Italy).

Matrices. Dried aerial parts of common O. basilicum L., $O$. vulgare L., and T. vulgaris L. samples were purchased from a local dealer; all matrices belonged to A. MINARDI \& FIGLI S.R.L. Company (Bagnacavallo, Italy) from 2015 cultivations.

MSPD Extraction. The sample was homogenized, by an agate mortar, with a C18-T bonded phase (PhenomenexTorrance, CA, USA) in a 1:3 ratio. The homogenization was carried out until the color and aspect of the mixture was uniform; then, the resultant blend was packed in a glass cartridge (i.d. $1 \mathrm{~cm}$, capacity $3 \mathrm{~mL}$ ), with two polytetrafluoroethylene (PTFE) retaining frits settled on the top and bottom of it. The packed cartridge was fixed to a vacuum manifold system (Phenomenex) and it was processed as follows: a preliminary washing step was performed with $1 \mathrm{~mL}$ of water/ methanol 90:10 solution; then the analytes were eluted with $0.5 \mathrm{~mL}$ of methanol. The eluate was then filtered with a $0.2 \mu \mathrm{m}$ PTFE syringe filter and analyzed by a UHPLC-MS/MS system.

UHPLC-MS/MS Analysis. LC-MS/MS analysis was performed by a UHPLC system Nexera XR (Shimadzu, Tokyo, Japan) coupled to a 4500 Qtrap mass spectrometer (Sciex, Toronto, ON, Canada) equipped with a heated ESI source (V-source). The ion source parameters were set as follows: negative ionization mode; ion spray voltage $-4.5 \mathrm{kV}$; air as nebulizer gas at 40 psi, nitrogen as turbo gas at 40 psi; and temperature at $500{ }^{\circ} \mathrm{C}$. An MRM acquisition mode, with two precursor ion/fragment ion transitions for each analyte, was carried out, in order to have the highest specificity.

For the chromatographic separation, an Excel 2 C18-PFP column $(2 \mu \mathrm{m}, 10 \mathrm{~cm} \times 2.1 \mathrm{~mm}$ ID-ACE, Aberdeen, UK) was used; it was assembled with a column saver, to avoid 
Table 3. Recoveries Calculated with the Standard Addition Method from Spiked O. basilicum L. (B), O. vulgare L. (O), and T. vulgaris L. (T), at Three Different Concentrations

\begin{tabular}{|c|c|c|c|c|c|c|c|c|c|}
\hline \multicolumn{10}{|c|}{ recoveries $\%($ RSD \%) $n=3$} \\
\hline \multirow[b]{2}{*}{ compound } & \multicolumn{3}{|c|}{ low } & \multicolumn{3}{|c|}{ medium } & \multicolumn{3}{|c|}{ high } \\
\hline & B & $\mathrm{T}$ & $\mathrm{O}$ & B & $\mathrm{T}$ & $\mathrm{O}$ & B & $\mathrm{T}$ & $\mathrm{O}$ \\
\hline gallic acid & $75(8)$ & $67(9)$ & $80(7)$ & $80(4)$ & $77(9)$ & $92(7)$ & $70(5)$ & $89(13)$ & $83(11)$ \\
\hline caftaric acid & $70(7)$ & $68(6)$ & $75(9)$ & $78(9)$ & $86(7)$ & $87(10)$ & $88(9)$ & $72(9)$ & $72(7)$ \\
\hline chlorogenic acid & $89(5)$ & $71(3)$ & $88(10)$ & $95(11)$ & $79(12)$ & $75(8)$ & $93(9)$ & $97(8)$ & $91(4)$ \\
\hline 4-OH benzoic acid & $78(12)$ & $67(11)$ & $75(11)$ & $83(5)$ & $80(5)$ & $75(9)$ & $79(5)$ & $68(11)$ & $79(11)$ \\
\hline 4-OH phenylacetic acid & $91(9)$ & $87(12)$ & $90(8)$ & $87(9)$ & $77(6)$ & $92(11)$ & $78(3)$ & $84(3)$ & $89(9)$ \\
\hline isoquercetin & $75(7)$ & $77(8)$ & $82(4)$ & $82(5)$ & $68(9)$ & $78(3)$ & $79(10)$ & $83(14)$ & $79(5)$ \\
\hline caffeic acid & $75(3)$ & $66(7)$ & $85(9)$ & $83(10)$ & $73(14)$ & $72(7)$ & $87(5)$ & $79(8)$ & $86(5)$ \\
\hline vanillic acid & $91(4)$ & $71(13)$ & $67(5)$ & $86(11)$ & $82(10)$ & $82(4)$ & $89(2)$ & $90(9)$ & $77(3)$ \\
\hline syringic acid & $82(3)$ & $66(5)$ & $75(7)$ & $89(3)$ & $73(11)$ & $70(9)$ & $77(5)$ & $83(11)$ & $85(5)$ \\
\hline$p$-coumaric acid & $86(5)$ & $72(9)$ & $90(10)$ & $89(9)$ & $89(12)$ & $92(10)$ & $79(3)$ & $80(4)$ & $88(10)$ \\
\hline ferulic acid & $83(9)$ & $65(9)$ & $70(7)$ & $91(5)$ & $94(11)$ & $89(8)$ & $87(4)$ & $78(10)$ & $91(4)$ \\
\hline chicoric acid & $87(3)$ & $74(8)$ & $88(6)$ & $79(11)$ & $80(12)$ & $76(11)$ & $77(7)$ & $71(11)$ & $102(7)$ \\
\hline rutin & $77(10)$ & $75(11)$ & $80(4)$ & $88(10)$ & $77(3)$ & $72(7)$ & $76(9)$ & $85(9)$ & $94(11)$ \\
\hline$o$-coumaric acid & $79(5)$ & $67(11)$ & $82(9)$ & $82(3)$ & $89(10)$ & $92(10)$ & $85(5)$ & $89(12)$ & $69(10)$ \\
\hline rosmarinic acid & $97(12)$ & $94(13)$ & $87(7)$ & $105(9)$ & $73(2)$ & $96(9)$ & $91(5)$ & $74(10)$ & $97(8)$ \\
\hline cinnamic acid & $90(9)$ & $88(9)$ & $78(5)$ & $88(5)$ & $69(10)$ & $76(4)$ & $82(8)$ & $90(13)$ & $93(10)$ \\
\hline quercetin & $78(10)$ & $67(8)$ & $84(9)$ & $86(4)$ & $79(8)$ & $89(9)$ & $88(4)$ & $98(7)$ & $72(4)$ \\
\hline
\end{tabular}

Table 4. PC Amounts in O. basilicum L. (B), O. vulgare L. (O), and T. vulgaris L. (T)

\begin{tabular}{|c|c|c|c|}
\hline \multicolumn{4}{|c|}{ average amount (RSD \%) $\left(\mathrm{mg} \mathrm{g}^{-1}\right)$} \\
\hline compound & B & $\mathrm{T}$ & $\mathrm{O}$ \\
\hline gallic acid & $<$ LOQ & $0.98(9)$ & $\mathrm{n} / \mathrm{d}$ \\
\hline caftaric acid & $3.69(10)$ & $2.10(10)$ & $2.85(2)$ \\
\hline chlorogenic acid & $0.03(6)$ & $0.98(12)$ & $0.62(7)$ \\
\hline 4-OH benzoic acid & $0.64(14)$ & $1.62(7)$ & $0.87(2)$ \\
\hline 4-OH phenylacetic acid & $2.18(4)$ & $2.57(5)$ & $3.19(9)$ \\
\hline isoquercetin & $5.30(13)$ & $2.37(5)$ & $6.54(5)$ \\
\hline caffeic acid & $3.24(11)$ & $5.72(9)$ & $1.56(9)$ \\
\hline vanillic acid & $3.25(7)$ & $5.36(9)$ & $1.47(10)$ \\
\hline syringic acid & $0.62(5)$ & $2.67(9)$ & $0.35(6)$ \\
\hline$p$-coumaric acid & $3.33(5)$ & $3.59(12)$ & $2.13(8)$ \\
\hline ferulic acid & $2.31(9)$ & $5.38(7)$ & $1.52(11)$ \\
\hline chicoric acid & $14.60(12)$ & $8.76(6)$ & $9.65(12)$ \\
\hline rutin & $4.26(10)$ & $1.04(6)$ & $5.86(11)$ \\
\hline$o$-coumaric acid & $0.14(8)$ & $0.31(7)$ & $0.86(4)$ \\
\hline rosmarinic acid & $7.91(10)$ & $13.95(10)$ & $8.94(11)$ \\
\hline cinnamic acid & $2.37(8)$ & $1.75(9)$ & $2.95(6)$ \\
\hline quercetin & $0.04(3)$ & $0.20(10)$ & $<\mathrm{LOQ}$ \\
\hline
\end{tabular}

contaminants and microparticulate damages. The mobile phases were: (A) aqueous $0.1 \%$ formic acid and (B) acetonitrile. The flow rate was $0.3 \mathrm{~mL} \mathrm{~min}^{-1}$, driven completely into the V-source. For the effective separation of the analytes, a gradient profile was employed: $5 \%$ phase B was increased up to $100 \%$ in $5 \mathrm{~min}$, held for $1 \mathrm{~min}$, and switched back to $5 \%$ in $3 \mathrm{~min}$ (total time $6 \mathrm{~min}$ ). ${ }^{28}$

The quantitation of the analytes was achieved with the standard addition method and the peak areas of the selected ions were defined using Sciex MultiQuant software.

The quantifier transitions of each analyte, together with the main UHPLC-MS/MS parameters, are reported in Table 5, whereas the extracted ion currents (XICs) for all target analytes are reported in Figure 3.
Table 5. UHPLC-MS/MS Parameters for Each Phenolic Compound

\begin{tabular}{|c|c|c|c|c|c|c|c|}
\hline compound & $\left(\begin{array}{c}t_{\mathrm{rit}} \\
(\mathrm{min})\end{array}\right.$ & $\begin{array}{c}Q_{1} \\
(\mathrm{amu})\end{array}$ & $\begin{array}{l}\text { DP } \\
(\mathrm{V})\end{array}$ & $\begin{array}{c}\text { EP } \\
(V)\end{array}$ & $\begin{array}{c}Q_{3} \\
(\mathrm{amu})\end{array}$ & $\begin{array}{l}\text { CE } \\
(\mathrm{V})\end{array}$ & $\begin{array}{c}\text { CXP } \\
(\mathrm{V})\end{array}$ \\
\hline gallic acid & 2.51 & 169.0 & -51 & -10 & 125.0 & -20 & -4 \\
\hline caftaric acid & 3.76 & 311.0 & -129 & -4 & 148.9 & -14 & -5 \\
\hline chlorogenic acid & 3.79 & 353.1 & -14 & -11 & 191.0 & -23 & -6 \\
\hline $\begin{array}{l}\text { 4-OH benzoic } \\
\text { acid }\end{array}$ & 3.87 & 137.0 & -9 & -13 & 93.0 & -18 & -6 \\
\hline $\begin{array}{l}\text { 4-OH } \\
\text { phenylacetic } \\
\text { acid }\end{array}$ & 3.88 & 151.0 & -37 & -13 & 107.0 & -10 & -3 \\
\hline isoquercetin & 3.94 & 463.0 & -120 & -10 & 300.0 & -30 & -18 \\
\hline caffeic acid & 4.10 & 179.0 & -50 & -5 & 135.0 & -21 & -5 \\
\hline vanillic acid & 4.11 & 167.0 & -50 & -6 & 151.3 & -18 & -5 \\
\hline syringic acid & 4.26 & 197.0 & -44 & -10 & 120.8 & -23 & -8 \\
\hline$p$-coumaric acid & 4.47 & 162.6 & -29 & -6 & 119.0 & -19 & -8 \\
\hline ferulic acid & 4.61 & 193.0 & -52 & -9 & 134.0 & -21 & -4 \\
\hline chicoric acid & 4.70 & 473.0 & -35 & -9 & 149.0 & -28 & -9 \\
\hline rutin & 4.76 & 609.4 & -35 & -10 & 301.0 & -45 & -5 \\
\hline$o$-coumaric acid & 4.83 & 162.6 & -29 & -6 & 119.0 & -19 & -8 \\
\hline rosmarinic acid & 4.84 & 359.1 & -15 & -10 & 160.9 & -22 & -6 \\
\hline cinnamic acid & 5.19 & 146.9 & -46 & -13 & 77.1 & -31 & -5 \\
\hline quercetin & 5.37 & 301.0 & -39 & -10 & 151.0 & -24 & -14 \\
\hline
\end{tabular}

Validation Parameters. The validation parameters assessed were matrix effect, linearity, precision, recovery, LOQs, and LODs.

For each analyte, a calibration curve was built with 11 points, repeated in triplicate $\left(0.1-250 \mathrm{ng} \mathrm{mL}{ }^{-1}\right)$ of PC standard solutions. For each concentration level, injections were performed in triplicate and the average value was used for the external standard calibration curves.

Because of the absence of "blank" matrices, matrix effect ion suppression was quantitatively evaluated as proposed by Faccin et al. ${ }^{29}$ by the following equation 


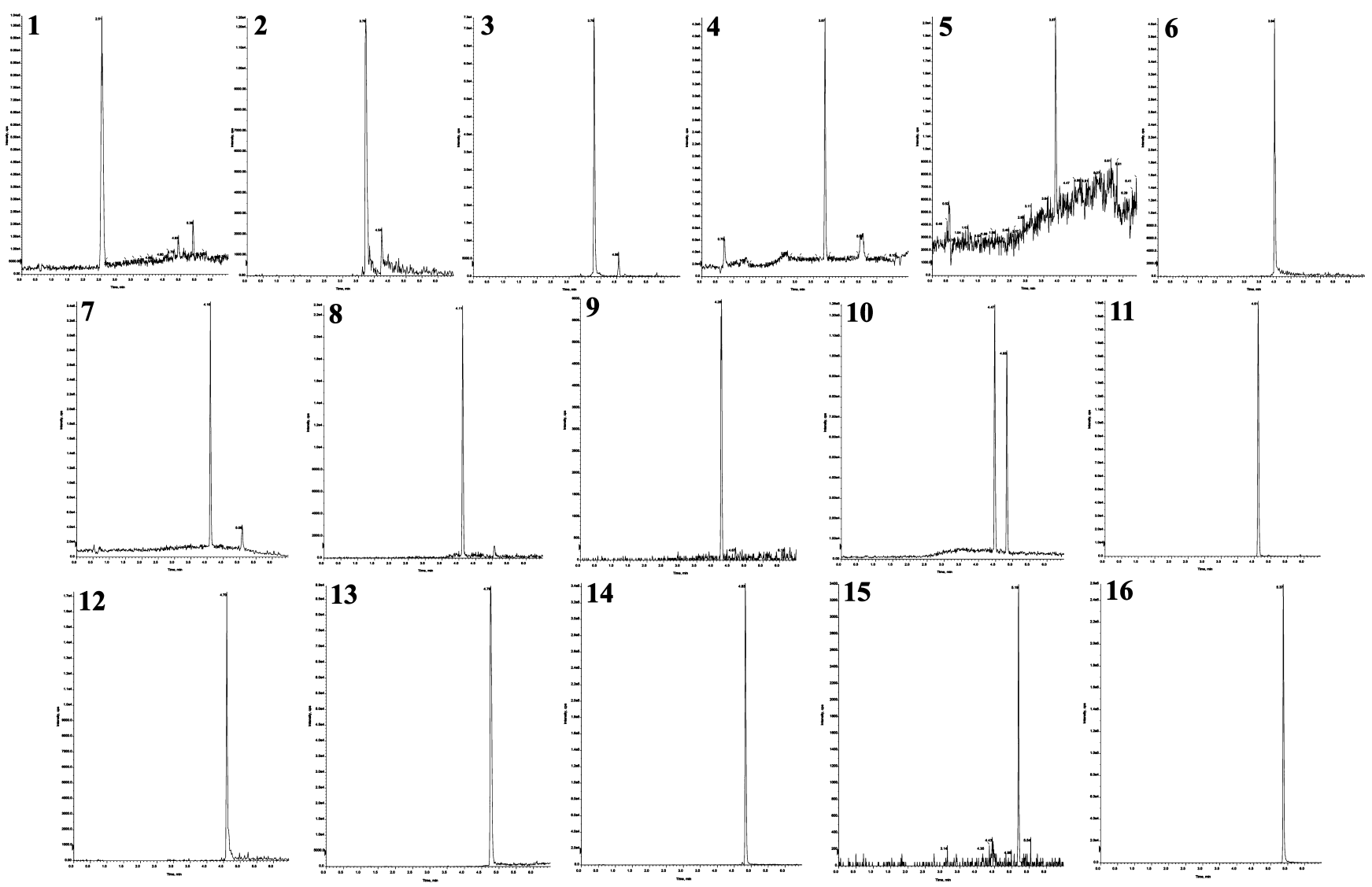

Figure 3. XICs for the target phenolic compounds. In the figure: 1-gallic acid; 2-caftaric acid; 3-chlorogenic acid; 4-4-OH phenylacetic acid; 5-4-OH benzoic acid; 6- caffeic acid; 7-vanillic acid; 8-syringic acid; 9-chicoric acid; $10-p$-coumaric acid and $o$-coumaric acid; $11-$ ferulic acid; 12-rutin; 13-rosmarinic acid; 15-cinnamic acid; 16-quercetin.

Ion suppression $(\%)$

$$
=\left\lceil\frac{\text { area }_{\text {spiked extracts }- \text { area }_{\text {matrix }}}}{\text { area }_{\text {std }}}-1\right\rceil \times 100
$$

Furthermore, the qualitative evaluation of matrix effect ion suppression was evaluated by a post-column infusion system coupled to the UHPLC system through a T connection. Thus, the MSPD extract was injected along with a continuous infusion $\left(10 \mu \mathrm{L} \mathrm{min}{ }^{-1}\right)$ of analytes' solution $\left(1 \mathrm{mg} \mathrm{L}^{-1}\right)$. The mobile phase injection into the chromatographic system allowed to obtain the reference baseline. Each analyte's transitions were monitored in the MRM mode in the MS/ MS system. In this way, the signal intensity decreases with respect to the baseline, designated matrix components responsible for ion suppression. ${ }^{30}$

Recovery values were found by spiking a known amount of sample with standard solution at three different concentration levels for each PC, starting from its natural content in these plant samples. The recovery calculations were carried out by the following equation

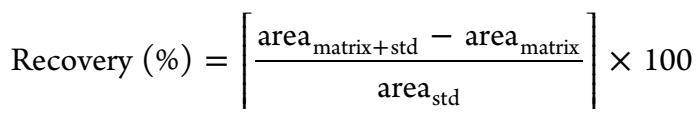

The LODs and LOQs were obtained at the signal-to-noise ratios of $3: 1$ and 10:1, respectively, by analyzing different diluted standard samples.
For intraday RSDs were considered one-day measures of six sample replicates (intraday precision or repeatability), whereas for interday RSDs, samples were analyzed for three consecutive days and each day twice (interday precision or withinlaboratory reproducibility).

\section{ASSOCIATED CONTENT}

\section{Supporting Information}

The Supporting Information is available free of charge on the ACS Publications website at DOI: 10.1021/acsomega.8b02239.

XICs of blank, O. basilicum L. matrix, and phenolic compounds (PDF)

\section{AUTHOR INFORMATION}

\section{Corresponding Author}

*E-mail: msergi@unite.it. Phone: +390861266949. Fax: +390861266915 (M.S.).

\section{ORCID}

Manuel Sergi: 0000-0002-1130-5294

\section{Author Contributions}

M.C.S. and M.P. contributed equally. The article was written through contributions of all the authors. All the authors have given approval to the final version of the article.

\section{Notes}

The authors declare no competing financial interest. 


\section{ABBREVIATIONS}

MSPD, matrix solid-phase dispersion; UHPLC-MS/MS, ultra-high-performance liquid chromatography coupled to tandem mass spectrometry

\section{REFERENCES}

(1) Azmir, J.; Zaidul, I. S. M.; Rahman, M. M.; Sharif, K. M.; Mohamed, A.; Sahena, F.; Jahurul, M. H. A.; Ghafoor, K.; Norulaini, N. A. N.; Omar, A. K. M. Techniques for extraction of bioactive compounds from plant materials: A review. J. Food Eng. 2013, 117, 426-436.

(2) Popa, V. I.; Dumitru, M.; Volf, I.; Anghel, N. Lignin and polyphenols as allelochemicals. Ind. Crops Prod. 2008, 27, 144-149.

(3) Naczk, M.; Shahidi, F. Phenolics in cereals, fruits and vegetables: Occurrence, extraction and analysis. J. Pharm. Biomed. Anal. 2006, 41, $1523-1542$

(4) Ignat, I.; Volf, I.; Popa, V. I. A critical review of methods for characterisation of polyphenolic compounds in fruits and vegetables. Food Chem. 2011, 126, 1821-1835.

(5) Petersen, M. Rosmarinic acid. Phytochemistry 2003, 62, 121125.

(6) Scalbert, A.; Johnson, I. T.; Saltmarsh, M. Polyphenols: antioxidants and beyond. Am. J. Clin. Nutr. 2005, 81, 215S-217S.

(7) Della Pelle, F.; Compagnone, D. Nanomaterial-based sensing and biosensing of phenolic compounds and related antioxidant capacity in food. Sensors 2018, 18, 462.

(8) Della Pelle, F.; González, M. C.; Sergi, M.; Del Carlo, M.; Compagnone, D.; Escarpa, A. Gold Nanoparticles-based ExtractionFree Colorimetric Assay in Organic Media: An Optical Index for Determination of Total Polyphenols in Fat-Rich Samples. Anal. Chem. 2015, 87, 6905-6911.

(9) Della Pelle, F.; Vilela, D.; González, M. C.; Lo Sterzo, C.; Compagnone, D.; Del Carlo, M.; Escarpa, A. Antioxidant capacity index based on gold nanoparticles formation. Application to extra virgin olive oil samples. Food Chem. 2015, 178, 70-75.

(10) Dai, J.; Mumper, R. J. Plant Phenolics: Extraction, Analysis and Their Antioxidant and Anticancer Properties. Molecules 2010, 15, 7313-7352.

(11) Carbone, K.; Giannini, B.; Picchi, V.; Lo Scalzo, R.; Cecchini, F. Phenolic composition and free radical scavenging activity of different apple varieties in relation to the cultivar, tissue type and storage. Food Chem. 2011, 127, 493-500.

(12) Javanmardi, J.; Khalighi, A.; Kashi, A.; Bais, H. P.; Vivanco, J. M. Chemical characterization of basil (Ocimum basilicum L.) found in local accessions and used in traditional medicines in Iran. J. Agric. Food Chem. 2002, 50, 5878-5883.

(13) Jayasinghe, C.; Gotoh, N.; Aoki, T.; Wada, S. Phenolics composition and antioxidant activity of sweet basil (Ocimum basilicum L.). J. Agric. Food Chem. 2003, 51, 4442-4449.

(14) Fang, Z.; Zhang, M.; Wang, L. HPLC-DAD-ESIMS analysis of phenolic compounds in bayberries (Myrica rubra Sieb. et Zucc.). Food Chem. 2007, 100, 845-852.

(15) Omoba, O.; Obafaye, R.; Salawu, S.; Boligon, A.; Athayde, M. HPLC-DAD Phenolic Characterization and Antioxidant Activities of Ripe and Unripe Sweet Orange Peels. Antioxidants 2015, 4, 498-512.

(16) Rohloff, J. Analysis of phenolic and cyclic compounds in plants using derivatization techniques in combination with GC-MS-based metabolite profiling. Molecules 2015, 20, 3431-3462.

(17) Al-Owaisi, M.; Al-Hadiwi, N.; Khan, S. A. GC-MS analysis, determination of total phenolics, flavonoid content and free radical scavenging activities of various crude extracts of Moringa peregrina (Forssk.) Fiori leaves. Asian Pac. J. Trop. Biomed. 2014, 4, 964-970.

(18) Lee, J.; Scagel, C. F. Chicoric acid found in basil (Ocimum basilicum L.) leaves. Food Chem. 2009, 115, 650-656.

(19) Wen, D.; Li, C.; Di, H.; Liao, Y.; Liu, H. A Universal HPLC Method for the Determination of Phenolic Acids in Compound Herbal Medicines. J. Agric. Food Chem. 2016, 53, 6624-6629.
(20) Flamini, R.; De Rosso, M.; Bavaresco, L. Study of Grape Polyphenols by Liquid Chromatography-High-Resolution Mass Spectrometry (UHPLC/QTOF) and Suspect Screening Analysis. J. Anal. Methods Chem. 2015, 2015, 350259.

(21) Melliou, E.; Zweigenbaum, J. A.; Mitchell, A. E. Ultrahighpressure liquid chromatography triple-quadrupole tandem mass spectrometry quantitation of polyphenols and secoiridoids in california-style black ripe olives and dry salt-cured olives. J. Agric. Food Chem. 2015, 63, 2400-2405.

(22) Barker, S. A. Matrix solid phase dispersion (MSPD). J. Biochem. Biophys. Methods 2007, 70, 151-162.

(23) Capriotti, A. L.; Cavaliere, C.; Foglia, P.; Samperi, R.; Stampachiacchiere, S.; Ventura, S.; Laganà, A. Recent advances and developments in matrix solid-phase dispersion. Trends Anal. Chem. 2015, 71, 186-193.

(24) Benabderrahmane, W.; Lores, M.; Lamas, J. P.; Benayache, S. Matrix solid-phase dispersion as a tool for phytochemical and bioactivities characterisation: Crataegus oxyacantha L._A case study. Nat. Prod. Res. 2017, 35, 1220-1223.

(25) Rashidipour, M.; Heydari, R.; Feizbakhsh, A.; Hashemi, P. Rapid monitoring of carvacrol in plants and herbal medicines using matrix solid-phase dispersion and gas chromatography flame ionisation detector. Nat. Prod. Res. 2014, 29, 621-627.

(26) Sergi, M.; Gentili, A.; Perret, D.; Marchese, S.; Materazzi, S.; Curini, R. MSPD Extraction of Sulphonamides from Meat followed by LC Tandem MS Determination. Chromatographia 2007, 65, 757761

(27) Peng, L.-Q.; Li, Q.; Chang, Y.-x.; An, M.; Yang, R.; Tan, Z.; Hao, J.; Cao, J.; Xu, J.-J.; Hu, S.-S. Determination of natural phenols in olive fruits by chitosan assisted matrix solid-phase dispersion microextraction and ultrahigh performance liquid chromatography with quadrupole time-of-flight tandem mass spectrometry. J. Chromatogr. A 2016, 1456, 68-76.

(28) Chaves-López, C.; Usai, D.; Donadu, M. G.; Serio, A.; González-Mina, R. T.; Simeoni, M. C.; Molicotti, P.; Zanetti, S.; Pinna, A.; Paparella, A. Potential of Borojoa patinoi Cuatrecasas water extract to inhibit nosocomial antibiotic resistant bacteria and cancer cell proliferation in vitro. Food Funct. 2018, 9, 2725-2734.

(29) Faccin, H.; Viana, C.; do Nascimento, P. C.; Bohrer, D.; de Carvalho, L. M. Study of ion suppression for phenolic compounds in medicinal plant extracts using liquid chromatography - electrospray tandem mass spectrometry. J. Chromatogr. A 2016, 1427, 111-124.

(30) Furey, A.; Moriarty, M.; Bane, V.; Kinsella, B.; Lehane, M. Ion suppression; A critical review on causes, evaluation, prevention and applications. Talanta 2013, 115, 104-122. 